\title{
Prenatal Diagnosis and Management of Fetal Goiter: A Case Series from Tertiary Perinatal Center in South India
}

\author{
Manjula Budidapadu ${ }^{1}$, Suseela Vavilala², Geeta Kolar ${ }^{3}$
}

\begin{abstract}
Aims and objectives: The reported incidence of fetal goiter is 1 in 40,000 live births. The effects of fetal goiter and thyroid dysfunction are significant. The objective of our study is to assess the safety, feasibility, and efficacy of intra-amniotic instillation of levothyroxine (LT4) in fetal goitrous hypothyroidism.

Materials and methods: A retrospective observational study of prenatally diagnosed cases of fetal goiter on routine antenatal ultrasound between January 2010 and January 2018 at Fernandez Hospital Foundation, Hyderabad, tertiary perinatal referral center. Data retrieved from electronic database.

Results: During this period, 9 cases of fetal goiter were diagnosed on routine ultrasonography with the incidence of 1 in 7,000 live births. The gestation age at diagnosis ranged between 21 weeks and 29 weeks (mean 26 weeks). Of the nine fetuses with goiter, two were excluded as one ended in a miscarriage at 22 weeks and the other was terminated elsewhere at 26 weeks. Data were analyzed in the remaining seven fetuses. Cordocentesis was performed in five fetuses and amniocentesis was performed in one woman with dichorionic diamniotic (DCDA) twin gestation which revealed hypothyroid status. Fetal therapy was offered to all the remaining seven fetuses with intra-amniotic instillation of LT4 100 to $200 \mu \mathrm{g}$ at 2-3 weeks' interval. First dose of LT4 was given at mean gestation of 27 weeks (range 23-30 weeks), and the mean time between the identification of fetal goiter and the first dose of LT4 was 6 days (range 2-15 days). Number of injections given ranged between 1 and 7. No adverse events were recorded. Follow-up sonogram showed subjective reduction in the goiter size. All the babies had hypothyroidism at birth. Conclusion: Our case series confirms the safety and feasibility of intra-amniotic instillation of LT4 for fetal goitrous hypothyroidism. Although fetal therapy has resulted in reduction in the size of the goiter, the thyroid hormone levels at birth were low. Further research is required to establish the proper management guidelines for this disorder.
\end{abstract}

Keywords: Congenital hypothyroidism, Cord blood thyroid-stimulating hormone, Fetal goiter, Fetal therapy, Intra-amniotic levothyroxine. International Journal of Infertility and Fetal Medicine (2019): 10.5005/jp-journals-10016-1187

\section{INTRODUCTION}

Fetal goiter is uncommon with a reported incidence of 1 in 40,000 live births, yet can cause serious problem. ${ }^{1}$ It is defined as a fetal thyroid gland diameter more than 95th percentile for the period of gestation. ${ }^{2-4}$ Careful routine antenatal ultrasound (USG) screening can diagnose fetal goiter, which usually appears as symmetrical homogeneous mass in front of the neck. Moreover, hyperextension of neck and associated polyhydramnios makes the identification of fetal goiter relatively easy. ${ }^{5}$ Prenatal detection of this condition was first reported in $1980 .^{6}$

The causes of fetal goiter are maternal factors like deficiency or excess of iodine, autoimmune thyroid disorders, exposure to medications or goitrogens which can cross placenta and affect fetal thyroid function, and congenital metabolic disorders of thyroid function. ${ }^{7-10}$

In maternal autoimmune thyroid disorders, thyroid-stimulating hormone (TSH) autoantibodies can cross placenta and stimulate fetal thyroid gland resulting in a goitrous hyperthyroidism, which can present with fetal tachycardia and subsequently develop fetal hydrops. Increased levels of maternal antithyroid peroxidase (antiTPO) and antithyroglobulin titers may lead to the suppression of thyroid gland and goitrous hypothyroidism in the fetus. Furthermore, maternal antithyroid treatment may inhibit fetal thyroid peroxidase. ${ }^{11}$

Blockage of fetal thyroid hormone synthesis causes increase in fetal TSH levels, which leads to the enlargement of fetal thyroid gland resulting in fetal goiter. This enlarged fetal thyroid
${ }^{1}$ Department of Obstetrics and Gynecology, Fernandez Hospital, Hyderabad, Telangana, India

2,3 Department of Fetal Medicine, Fernandez Hospital Foundation, Hyderabad, Telangana, India

Corresponding Author: Manjula Budidapadu, Department of Obstetrics and Gynecology, Fernandez Hospital, Hyderabad, Telangana, India, Phone: +91 40 41411215, e-mail: drmanjula. budidapadu@fernandezhospital.foundation

How to cite this article: Budidapadu M, Vavilala S, Kolar G. Prenatal Diagnosis and Management of Fetal Goiter: A Case Series from Tertiary Perinatal Center in South India. Int J Infertil Fetal Med 2019;10(3):37-41.

Source of support: Nil

Conflict of interest: None

gland causes compressive effect on the esophagus and tracheal tube, which can lead to excessive of amniotic fluid, fetal neck hyperextension, and labor dystocia. ${ }^{12}$ At birth, fetal goiter may cause tracheal obstruction leading to fetal asphyxia and demise. Therefore, prenatal detection of fetal goiter is very important for the immediate postnatal management of such fetuses at birth. In few affected infants, there was also reported psychomotor retardation in the later stages of life. ${ }^{13}$

Fetal goiter can be of hypothyroid/hyperthyroid status and therefore to determine fetal thyroid status direct measurement of thyroid hormone levels in amniotic fluid or cord blood samples 
should be performed. ${ }^{14,15}$ The gold standard for the diagnosis is fetal cordocentesis, but it is associated with $1 \%$ risk of fetal demise, even in skilled hands ${ }^{16}$ and the fetal therapy requires intra-amniotic instillation of levothyroxine (LT4), as the transplacental transfer of thyroxine is limited. ${ }^{17}$

Monitoring the effectiveness of fetal therapy is still a difficulty as it involves repeated fetal blood sampling (FBS), which is associated with very high fetal demise rates. Therefore, monitoring the effectiveness of fetal therapy should be performed by serial USG follow-up by looking at the size of fetal goiter instead of repeated FBS. $^{10}$

With this background, we investigated our case series of fetal goiter. The aim of our study was to analyze our cases of antenatally diagnosed and managed cases of fetal goiter and the neonatal outcome.

\section{Materials and Methods}

A retrospective study of all fetal goiter cases diagnosed from January 2010 to January 2018 at Fernandez Hospital Foundation, a tertiary perinatal center, Hyderabad, India was performed. We have included all cases of fetal goiter in singleton and multiple pregnancies.

We have compared measurements of fetal thyroid gland with the gestational age (GA)-specific nomograms. Goiter was defined as a thyroid gland volume equal to or greater than the mean +2 $\mathrm{SD}^{2}$ or as a thyroid gland diameter equal to or greater than the 95th percentile. ${ }^{4}$ We have noted the GA at the time of diagnosis of fetal goiter and levels of thyroid hormone and TSH in fetal blood or amniotic fluid sample, measured soon after the detection of fetal goiter. We have compared these levels with the reference values established by Guibourdenche et al. ${ }^{14}$ for fetal blood and by Singh et al. ${ }^{15}$ for amniotic fluid. If fetuses found to be hypothyroid, treatment was offered with intra-amniotic instillation of LT4 100-200 $\mu \mathrm{g}$ at 2-3 weeks' interval and we have collected the following details: fetal heart rate, fetal growth, amount of amniotic fluid, the number and frequency and dosage of LT4 injections and GA at initiation of therapy, measurements of fetal thyroid gland, and hormone levels before and after the fetal therapy. We have taken a written informed consent from all the mothers before starting the fetal therapy. We have performed physical examination and USG neck for thyroid gland size of these neonates at birth. Thyroid hormone and TSH levels were measured in cord blood at birth to assess the efficacy of fetal therapy. We have compared these levels with the established values by Guibourdenche et al. ${ }^{14}$
Data retrieval was from electronic medical records and astraia software. The follow-up of the survivors was performed through telephonic interview of parents.

\section{RESULTS}

During this period, 9 cases of fetal goiter were diagnosed on routine ultrasonography with the incidence of 1 in 7,000 live births.

\section{Maternal History}

[Eight mothers-there was one case of dichorionic diamniotic (DCDA) twin gestation]

Of the eight mothers, five were euthyroid, two were hyperthyroid [one on propylthiouracil (PTU) treatment, other on block replacement therapy-PTU and LT4], and the remaining one was hypothyroid with positive antithyroid antibodies.

\section{Prenatal Diagnosis and Management}

Of the nine fetuses with goiter, two were excluded as one ended in a miscarriage at 22 weeks and in the other was terminated elsewhere at 26 weeks in view of associated severe early onset fetal growth restriction with oligohydramnios. Data were analyzed in the remaining seven fetuses.

The gestation age at diagnosis ranged between 21 weeks and 29 weeks (mean 26 weeks). A solid anterior neck mass with neck extension and polyhydramnios was visualized in all cases. Table 1 shows the date on goiter size and fetal thyroid functional tests.

We have measured TSH levels in the cord blood samples in five fetuses whereas in DCDA twins with in vitro fertilization (IVF) conception, TSH levels were measured in amniotic fluid sample, considering the high-risk factors. These levels revealed fetal hypothyroid status. Karyotype was normal in all the fetuses. Fetal therapy was offered to all the seven fetuses with intra-amniotic instillation of LT4 100-200 $\mu$ g at 2-3 weeks' interval (Table 2). First dose of LT4 was given at mean gestation of 27 weeks (range 23-30 weeks), and the mean time between the identification of fetal goiter and the first dose of LT4 was 6 days (range 2-15 days). Number of injections given ranged between 1 and 7 .

After prenatal treatment, follow-up sonograms were performed in all fetuses, which showed subjective reduction in the goiter size with normal fetal growth and heart rate. Follow-up cord blood TSH levels were performed in two fetuses, which showed a downward trend.

No adverse events of fetal therapy, such as infection or fetal loss, were noted.

Table 1: Ultrasound findings and laboratory data at detection of fetal goiter

\begin{tabular}{|c|c|c|c|c|c|}
\hline \multirow[b]{4}{*}{ Fetus } & \multirow{4}{*}{$\begin{array}{l}\text { Gestational age at } \\
\text { diagnosis }\end{array}$} & \multirow{4}{*}{$\begin{array}{l}\text { Size of goiter (diameter } \\
\text { in } \mathrm{mm} \text { ) }\end{array}$} & \multicolumn{3}{|c|}{ Fetal thyroid function tests } \\
\hline & & & TSH & T3 & $T 4$ \\
\hline & & & $N: 2.3-13$ & $N: 6-7$ & $N: 6-7$ \\
\hline & & & $\mathrm{mIU} / \mathrm{L}$ & $n g / m L$ & $\mu g / d L$ \\
\hline 1 & $29 w+5 d$ & $37 \times 33$ & $>100$ & & 0.36 \\
\hline 2 & $21 w+1 d$ & $25 \times 16$ & $>100$ & 0.5 & 3.72 \\
\hline 3 & $27+1$ & $28 \times 16$ & $>100$ & 0.37 & 1.88 \\
\hline \multirow[t]{2}{*}{$4,5^{*}$} & $25+6$ & $33 \times 22($ fetus $A)$ & $1.4^{\#}$ & & \\
\hline & & $38 \times 37$ (fetus B) & $2^{\#}$ & & \\
\hline 6 & $28+5$ & $36 \times 28$ & 30 & & \\
\hline 7 & $27+4$ & $35 \times 32$ & $>100$ & & \\
\hline
\end{tabular}

*Dichorionic diamniotic twin gestation

\#Normal levels of TSH in amniotic fluid: $0.06-0.2 \mathrm{mIU} / \mathrm{L}$. In the remaining fetuses cord blood sampling was performed 
Table 2: L-Thyroxine regimens

\begin{tabular}{llll}
\hline & \multirow{2}{*}{$\begin{array}{c}\text { GA at treatment } \\
\text { initiation (weeks) }\end{array}$} & No. of LT4 injections $(\mu \mathrm{g} / \mathrm{inj})$. & Dose \\
\cline { 3 - 4 } Fetus & 30 & $2(30,32)$ & $200+200$ \\
2 & 22 & $7(22,24,26,28,30,32,34)$ & $7(200)$ \\
3 & 28 & $4(28,30,32,34)$ & $200+100+100+100$ \\
$4,5^{*}$ & 28 & $2(28,31)$ in each sac & $200+100$ \\
6 & 32 & $3(32,34,36)$ & $200+200+200$ \\
7 & 28 & $1(28)$ & 200 \\
\hline
\end{tabular}

*Dichorionic diamniotic twin gestation

Table 3: Outcomes

\begin{tabular}{llllll}
\hline Fetus & $\begin{array}{l}\text { Gestational age at } \\
\text { delivery (weeks) }\end{array}$ & Outcome (APGAR) & Cord blood TSH & FT3 (pg/mL): N: 2-6 & FT4 (ng/dL): N: $1.3-1.4$ \\
\hline 1 & 37 & Live/F/2.8 kg & $>100$ & 1.89 & 0.4 \\
2 & $35+4$ & Live/M/2.9 kg & 25.8 & 5.2 & 8.07 \\
3 & 37 & Live/M/3.1 kg & $>100$ & 6 & 1.53 \\
$4,5^{*}$ & $36+1$ & Live/F/2.4 kg and & 15 & 0.5 & 8.07 \\
& & live/M/2.3 kg & & & \\
6 & $38+6$ & Live/M/3.1 kg & 20 & & \\
$7^{\#}$ & 30 & Live/F/1 kg & $>100$ & & \\
\hline
\end{tabular}

*Dichorionic diamniotic twin gestation

\#Had infant death at 2 months of age-prematurity related

\section{Delivery and Postnatal Details (Table 3)}

Of the seven fetuses treated, three had term deliveries and four had preterm deliveries ( 36 weeks - twins, 30 weeks, 35 weeks). One had infant death at 2 months of age (prematurity related). Postnatal neck examination showed slight enlargement of thyroid gland in five fetuses and USG neck revealed the same.

Cord blood TSH at birth showed hypothyroid status in all and tab. Levothyroxine oral (replacement therapy) was started. At last follow-up, all six babies were healthy and growing well with normal psychomotor development. Four babies are still on maintenance dose of LT4, remaining two babies are not requiring any maintenance dose as the causative factor (antithyroid drugPTU) is no more present.

\section{Discussion}

We can assess the fetal thyroid gland antenatally by USG from 20 to 36 weeks. ${ }^{2}$ The case reports on fetal goiter have been gradually increasing, although it is a rare condition, due to the advancements in USG technology. ${ }^{18}$ This was obviously evidenced from our case series with incidence of 1 in 7,000 which is quite higher than the reported incidence of 1 in 40,000. ${ }^{1}$

Fetal goiter should be differentiated from all other abnormalities in the front and lateral aspect of fetal neck, which includes cystic hygromas, teratomas, lymphangiomas/hemangiomas, branchial cleft cysts, thyroglossal cysts, and other developmental cystic lesions. ${ }^{19}$ We can differentiate these lesions from fetal goiter by their usual appearance of fluid-filled cystic masses. ${ }^{20}$

Of the eight mothers, five were euthyroid, two were hyperthyroid on PTU treatment, and the remaining one was hypothyroid with positive antithyroid antibodies. The cause of fetal goiter in euthyroid mothers may be due to congenital metabolic disorders of thyroid function. In a mother with hypothyroidism, antithyroid antibodies were positive, transplacental transfer of which might have caused fetal goiter. In mothers with hyperthyroidism who were on PTU treatment, cause might be due to transplacental transfer of PTU. In these mothers, optimal adjustments of PTU dosage might have prevented fetal goiter.

The detection of fetal thyroid gland enlargement on routine USG requires an appropriate diagnosis before starting the fetal therapy. Fetal goiter can be associated with hypo/hyperthyroid state. Hypothyroid goiter is common than hyperthyroid goiter, which has been confirmed in our case series. Huel et al. ${ }^{21}$ established a scoring system to distinguish between hypothyroid and hyperthyroid goiter on the basis of pattern of color Doppler, fetal heart rate, bone maturation, and fetal mobility. Score $<2$ suggests of hypothyroid and $\geq 2$ suggests of hyperthyroid goiter. However, we have not used this method as it is non-specific and inconsistent. In 1996, Abuhamad et al. ${ }^{22}$ recommended that cordocentesis can accurately assess the thyroid status of fetus. Thyroid-stimulating hormone does not have transplacental transfer, fetus secretes its own TSH. Therefore, the gold standard for the fetal thyroid status assessment is by cordocentesis. Gestational age-specific nomograms are available for fetal TSH levels. At any period of gestation, cord blood TSH levels $>12 \mathrm{mlU} / \mathrm{L}$ is suggestive of hypothyroid status, but fetal cordocentesis is associated with $1 \%$ risk of fetal demise, even in skilled hands. ${ }^{23}$ In cases where determination of serum fetal TSH levels by FBS may be risky, TSH levels in amniotic fluid can be measured for the management of fetal goiter. ${ }^{24}$ There is recent evidence showing that the fetal thyroid status can be reflected by TSH levels in amniotic fluid. ${ }^{25}$ In another report in 2005, Mirsaeid Ghazi et al. ${ }^{26}$ supported that amniotic fluid TSH levels could be used as a reliable measurement for the management of fetal goiter. Though the association between thyroid hormone and TSH levels in amniotic fluid and fetal blood 
remains uncertain, ${ }^{27}$ amniocentesis has been used for assessing fetal thyroid status as it is safe and easy than FBS.

Prenatal treatment includes intra-amniotic injections of LT4, which is a minimally invasive procedure to fetal treatment. The rational for prenatal treatment is to optimize fetal growth and development and to reduce goiter size, thereby relieving obstruction. The intra-amniotic instillation of LT4 improves fetal serum thyroid hormone levels in fetal goitrous hypothyroidism because the fetus swallows amniotic fluid in which LT4 is instilled. Dosage and frequency were based on the knowledge illustrated in the literature. ${ }^{22}$ Perelman et al. ${ }^{28}$ stated that fetal hypothyroid goiter was effectively managed intra-amniotic instillation of $500 \mu \mathrm{g}$ LT4 every 10-14 days. Davidson et al. ${ }^{12}$ has also reported that weekly intra-amniotic instillation of $250 \mu \mathrm{g}$ of LT4 would closely approximate the thyroxine requirement of a neonate with hypothyroidism ( $10 \mu \mathrm{g} / \mathrm{kg}$ body weight/day).

Weiner et al. ${ }^{6}$ first reported the antenatal diagnosis of fetal goiter in 1980 in a hyperthyroid mother using PTU. Barone et al. ${ }^{5}$ reported the second case in a euthyroid mother. They have managed cases successfully with cord blood sampling and intra-amniotic LT4 instillation. Till date around 30 cases of fetal goiter were reported in the literature. We can see that these numbers are quite a few to outline proper guidelines for optimal dosage and frequency of LT4 injection. 3D USG can be used for the evaluation and management of fetal goiters. ${ }^{29}$ The first report of 3D USG of fetal goiter was introduced in 2005. This technique allows the measurement of fetal thyroid volume and blood flow and monitoring the size of the goiter before and after the fetal therapy. ${ }^{29,30}$

Ours is a second case series of fetal goiter. Ribault et al. ${ }^{31}$ have published first case series of fatal goiter in 2009 which included 12 cases. In our case series, the gestation age at diagnosis ranged between 21 weeks and 29 weeks (mean 25 weeks) which is similar to the case series of Ribault et al. (21-32 weeks). Prenatal treatment included intra-amniotic installation of LT4. Mean GA at the first injection was little earlier (i.e., at 27 weeks, range 23-30) to that of Ribault et al. (29 weeks, range 24-36) and mean time between the detection of fetal goiter and the first dose of LT4 was 6 days (range 2-15), which is similar to as in Ribault et al. series (6 days, range 1-15). The dose per injection was 100-200 $\mu \mathrm{g}$ whereas in case series by Ribault et al. dose per injection varied widely from 200-800 $\mu \mathrm{g}$. Number of injections ranged from 1 to 7 which is similar to the earlier case series ${ }^{1-6}$ and frequency ranged between 1 week and 3 weeks, whereas in Ribault et al. it ranged between 1 week and 4 weeks. This variability reflects the lack of clear recommendations for management. The last injection was given on average 3.5 weeks before birth (range 1-5). In Ribault et al. case series, it was given 2.6 weeks before birth (range 1-11).

In our case series, no adverse events were recorded following prenatal treatment. It confirms the feasibility and safety of intraamniotic instillation of LT4 for fetal goitrous hypothyroidism. Moreover, a follow-up sonogram in all fetuses showed subjective reduction in the goiter size and follow-up cord blood TSH levels for thyroid function tests (TFTs) showed decreasing trend in three fetuses, suggesting the effect of treatment. In our case series, none of the fetuses had difficult delivery or tracheal compression at birth.

At birth, although there was reduction in the goiter size, thyroid hormone status remained low in all babies as was seen in case series by Ribault et al. Levothyroxine was started in all babies which may be due to suboptimal dosing regimens and the gap between last dose of intra-amniotic LT4 and delivery. Dudenhausen ${ }^{32}$ reported that the increase in plasma thyroid hormone levels after intraamniotic instillation of LT4 remained for only 5 days. Another factor may be due to differences in ingestion and intestinal absorption of amniotic fluid by the fetus. Combined analysis of all these factors may help in developing a proper treatment protocol.

As fetal goiter may be associated with chromosomal abnormalities especially T21, karyotyping was performed for all fetuses from amniotic fluid sample which showed normal. At last follow-up, all babies were healthy with normal growth and development.

In conclusion, prenatal diagnosis of fetal goiter requires a detailed maternal and fetal assessment. We have showed the prenatal detection and treatment of fetal goiter. Prenatal detection depends on a combination of USG, cordocentesis for initial assessment of fetal thyroid status, and follow-up by amniotic fluid TSH assay. Fetal therapy is given by intra-amniotic LT4 for fetal hypothyroidism. It reduces obstetric complications and promotes growth and intellectual development. However, infants may require lifelong replacement.

\section{References}

1. Corral E, Reascos M, Preiss $Y$, et al. Treatment of fetal goitrous hypothyroidism: Value of direct intramuscular L-thyroxine therapy. Prenat Diagn 2010;30(9):899-901. DOI: 10.1002/pd.2560.

2. Ho SS, Metreweli C. Normal fetal thyroid volume. Ultrasound Obstet Gynecol 1998;11(2):118-122.DOI: 10.1046/j.1469-0705.1998.11020118.x.

3. Achiron R, Rotstein Z, Lipitz S, et al. The development of the foetal thyroid: in utero ultrasonographic measurements. Clin Endocrinol (Oxf) 1998;48(3):259-264. DOI: 10.1046/j.1365-2265.1998.00388.x.

4. Ranzini AC, Ananth CV, Smulian JC, et al. Ultrasonography of the fetal thyroid: nomograms based on biparietal diameter and gestational age. JUltrasound Med 2001;20(6):613-617. DOI: 10.7863/ jum.2001.20.6.613.

5. Barone CM, Van Natta FC, Kourides IA, et al. Sonographic detection of fetal goitre, an unusual cause of hydramnios. J Ultrasound Med 1985;4(11):625-627. DOI: 10.7863/jum.1985.4.11.625.

6. Weiner S, Scharf J, Bolognese RJ, et al. Antenatal diagnosis and treatment of a fetal goitre. J Reprod Med 1980;24(1):39-42.

7. Glinoer $D$. The importance of iodine nutrition during pregnancy. Public Health Nutr 2007;10(12A):1542-1546. DOI: 10.1017/ S1368980007360886.

8. Polak M, Le Gac I, Vuillard E, et al. Fetal and neonatal thyroid function in relation to maternal Graves' disease. Best Pract Res Clin Endocrinol Metab 2004;18(2):289-302. DOI: 10.1016/j.beem.2004.03.009.

9. Caron P, Moya CM, Malet D, et al. Compound heterozygous mutations in the thyroglobulin gene (1143delC and 6725G-A [R2223H]) resulting in fetal goitrous hypothyroidism. J Clin Endocrinol Metab 2003; 8(8):3546-3553. DOI: 10.1210/jc.2002-021744.

10. Medeiros-Neto G, Bunduki V, Tomimori E, et al. Prenatal diagnosis and treatment of dyshormonogenetic fetal goitre due to defective thyroglobulin synthesis. J Clin Endocrinol Metab 1997;82(12):42394242. DOI: 10.1210/jcem.82.12.4407.

11. Rosenfeld H, Ornoy A, Shechtman S, et al. Pregnancy outcome, thyroid dysfunction and fetal goitre after in utero exposure to propylthiouracil: a controlled cohort study. Br J Clin Pharmacol 2009;68(4):609-617. DOI: 10.1111/j.1365-2125.2009.03495.x.

12. Davidson KM, Richards DS, Schatz DA, et al. Successful in utero treatment of fetalgoitre and hypothyroidism. N Engl J Med 1991;324(8):543-546. DOI: 10.1056/NEJM199102213240807.

13. New England Congenital Hypothyroidism Collaborative. Elementary school performance of children with congenital hypothyroidism. J Pediatr 1990;116(1):27-32. DOI: 10.1016/s0022-3476(05)81640-3.

14. Guibourdenche J, Noël M, Chevenne D, et al. Biochemical investigation of foetal and neonatal thyroid function using the 
ACS180SE analyser: clinical application. Ann Clin Biochem 2001;38(Pt 5):520-526. DOI: 10.1177/000456320103800509.

15. Singh PK, Parvin CA, Gronowski AM. Establishment of reference intervals for markers of fetal thyroid status in amniotic fluid. J Clin Endocrinol Metab 2003;88(9):4175-4179. DOI: 10.1210/jc.2003030522.

16. Grüner C, Kollert A, Wildt L, et al. Intrauterine treatment of fetal goitrous hypothyroidism controlled by determination of thyroidstimulating hormone in fetal serum. Fetal Diagn Ther 2001;16(1):4751. DOI: 10.1159/000053880.

17. Vulsma T, Gons MH, de Vijlder JJ. Maternal-fetal transfer of thyroxine in congenital hypothyroidism due to a total organification defect or thyroid agenesis. N Engl J Med 1989; 32(1):13-16. DOI: 10.1056/ NEJM198907063210103.

18. Meideros-Neto GA, Stanbury JB. Inherited disorders of the thyroid system. Boca Raton-CRC Pres 1994. 1-218.

19. Volumenie JL, Polak M, Guibourdenche J, et al. Managment of fetal goitres: a case report of 11 cases in a single perinatal unit. Prenat Diagn 2000;20(10):799-806. DOI: 10.1002/1097-0223(200010)20:103.0.co;2-v.

20. Farrell PT. Prenatal diagnosis and intrapartum management of neck masses causing airway obstruction. Paediatr Anaesth 2004;14(1):4852. DOI: $10.1046 /$ j.1460-9592.2003.01201.x.

21. Huel C, Guibourdenche J, Vuillard E, et al. Use of ultrasound to distinguish between fetal hyperthyroidism and hypothyroidism on discovery of a goiter. Ultrasound Obstet Gynecol 2009;33(4):412-420. DOI: 10.1002/uog.6315.

22. Abuhamad AZ, Fisher DA, Warsoff SL, et al. Antenatal diagnosis and treatment of fetal goitrous hypothyroidism: case report and review of the literature. Ultrasound ObstetGynecol 1995;6(5):368-371. DOI: 10.1046/j.1469-0705.1995.06050368.x.

23. Perrotin F, Sembely-Taveau C, Haddad G, et al. Prenatal diagnosis and early in utero management of fetal dyshormonogenetic goitre.
Eur J Obstet Gynecol Reprod Biol 2001;94(2):309-314. DOI: 10.1016/ s0301-2115(00)00346-8.

24. Baumann NA, Gronowski AM. Thyroid hormones in amniotic fluid. Am J Clin Pathol 2007;128(1):158-163. DOI: 10.1309/69A5AV266W230AUA.

25. Mayor-Lynn KA, Rohrs HJ, Cruz AC, et al. Antenatal diagnosis and treatment of a dyshormonogenetic fetal goitre. J Ultrasound Med 2009;28(1):67-71. DOI: 10.7863/jum.2009.28.1.67.

26. Mirsaeid Ghazi AA, Ordookhani A, Pourafkari M, et al. Intrauterine diagnosis and management of fetal goitrous hypothyroidism: a report of an Iranian family with three consecutive pregnancies complicated by fetal goitre. Thyroid. 2005;15(12):1341-1347. DOI: 10.1089/thy.2005.15.1341.

27. Francois $A$, Hindryck $x A$, Vandecruys $H$, et al. Fetal treatment for early dyshormonogenetic goitre. Prenat Diagn 2009;29(5):543-545. DOI: 10.1002/pd.2237.

28. Perelman AH, Johnson RL, Clemons RD, et al. Intrauterine diagnosis and treatment of fetal goitrous hypothyroidism. J Clin Endocrin Metab 1990;71(3):618-621. DOI: 10.1210/jcem-71-3-618.

29. Marin RC, Bello-Munoz JC, Martinez GV, et al. Use of 3-dimensional sonography for prenatal evaluation and follow-up of fetal goitrous hypothyroidism. J Ultrasound Med 2010;29(9):1339-1343. DOI: 10.7863/jum.2010.29.9.1339.

30. Nath CA, Oyelese $Y$, Yeo L, et al. Three-dimensional sonography in the evaluation and management of fetal goitre. Ultrasound Obstet Gynecol 2005;25(3):312-314. DOI: 10.1002/uog.1863.

31. Ribault V, Castanet $M$, Bertrand $A M$, et al. Experience with intraamniotic thyroxine treatment in nonimmune fetal goitrous hypothyroidism in 12 cases. J Clin Endocrinol Metab October;94(10):3731-3739. DOI: 10.1210/jc.2008-2681.

32. Dudenhausen JW. Alternatives to the antenatal glucocorticoid treatment for the prevention of respiratory distress syndrome. $J$ Perinat Med 1987;15(5):453-459. DOI: 10.1515/jpme.1987.15.5.453. 